\title{
Wohn- und Obdachlosigkeit
}

\section{Aktuelle Theorien in der angelsächsischen Forschung}

In der Diskussion um Wohn- und Obdachlosigkeit geht es nicht zuletzt auch darum, wie die jeweiligen Zustände definiert werden - nicht zuletzt, um passende Unterstützungsangebote zu machen. Einige Nationalstaaten weichen von den Festlegungen auf europäischer Ebene ab. Wie wird Wohnungslosigkeit im Vereinigten Königreich wissenschaftlich diskutiert?

$\mathrm{D}$ ie weithin akzeptierte Europäische Typologie der Wohnungslosigkeit (ETHOS) ${ }^{1}$ unterscheidet zwischen vier weit gefasste Situationen von Obdachlosigkeit und Gefährdung durch Wohnungslosigkeit:

- Obdachlosigkeit (Schlafen auf öffentlichen Plätzen),

- Wohnungslosigkeit (Aufenthalt in Notunterkünften),

- unsichere Wohnverhältnisse (wie z. B. Couchsurfing oder Leben in häuslicher Gewalt) und

- physisch unzureichende Wohnverhältnisse (Edgar et al. 2003).

In vielen Ländern ist die gesetzliche Definition jedoch wesentlich enger gefasst und beschränkt sich in der Regel auf die ersten beiden ETHOS-Kategorien, „Obdachlosigkeit“ und „Wohnungslosigkeit“. In der Republik Irland beispielsweise ist die gesetzliche Definition in Abschn. 2 des Housing Act 1988 zu finden. Dort gilt eine Person als obdachlos, wenn die lokale Behörde feststellt, dass die Person keine Unterkunft hat, in der sie angemessen wohnen kann, oder wenn die Person in einer Art Notunterkunft lebt und davon ausgegangen wird, dass die sie nicht über genügend Mittel verfügt, eine

Joe Finnerty
Cork, Irland
Studium der Sozialpolitik, Studienkoordinator der Abteilung für
angewandte Sozialstudien der University College Cork.
j.finnerty@ucc.ie

Zusammenfassung Der Beitrag beginnt mit einer Diskussion der aktuellen Theorie und Forschungzum Problembereich Obdachlosigkeit. Anschließend werden die verschiedenen Aspekte der Nutzerbeteiligung im Lichte dieser unterschiedlichen Dimensionen von Wohnungslosigkeit und Wohnungsprekarität untersucht. Er schließt mit einem Überblick über die aktuelle Forschung auf diesem Gebiet.

Schlüsselwörter Obdachlosigkeit, Wohnungslosigkeit, UK, Housing first, Lösungsansätze, Partizipation angemessene Unterkunft zu sichern. Obwohl dies ein breites Spektrum von Wohnungsbedürfnissen umfassen könnte, wird die Definition in der Praxis eng ausgelegt und konzentriert sich auf Personen, die auf der Straße schlafen und in Not- und Übergangsunterkünften leben (Daly 2019).

In den letzten Jahren hat sich ein akademischer Konsens in vier Bereichen der Theorie und Forschung herausgebildet - dies mit unterschiedlichen Auswirkungen auf die Obdachlosenpolitik und -praxis in vielen Ländern der EU und anderswo. Erstens ist unter den Obdachlosenforschern ein Konsens über die Ursachen von Obdachlosigkeit („Obdachlosigkeit“ und „Wohnungslosigkeit“) entstanden, der auf Arbeiten von Wissenschaftlern wie Wright et al. (1998), Fitzpatrick (2005), Busch-Geertsema et al. (2010) sowie Bramley und Fitzpatrick (2018) beruht. Im Großen und Ganzen sieht dieser Rahmen die Ursachen für Obdachlosigkeit in einer Kombination aus strukturellen, familiären und individuellen Faktoren, wobei die dominante Kombination Gegenstand empirischer Untersuchungen ist.

In dieser komplexen Mischung aus Armut, ungünstigen Kindheitserfahrungen, niedrigem Bildungsabschluss, Prekarität auf dem Arbeits- und Wohnungsmarkt sowie psychischer Gesundheit und Sucht- und Verhaltensproblemen stellt sich Wohnungslosigkeit als „das Ergebnis einer dynamischen Interaktion zwischen individuellen Merkmalen und Handlungen und strukturellen Veränderungen " dar (Busch-Geertsema et al. 2010, S. 5). Insgesamt ist das Risiko der Wohnungslosigkeit bei armen Haushalten, die von einem oder mehreren dieser zusätzlichen Risikofaktoren betroffen sind, hoch (Bramley und Fitzpatrick 2018). Darüber hinaus wurde die Hypothese aufgestellt, dass es einen umgekehrten Zusammenhang zwischen der Anzahl der wohnungslosen Bevölkerung und dem Grad der psychischen Gesundheit, Suchtproblemen und anderen Belastungen innerhalb dieser Bevölkerungsgruppe gibt (Stephens et al. 2010). 
Das zweite zentrale Forschungsergebnis bezieht sich auf eine Differenzierung innerhalb der wohnungslosen Bevölkerung nach der Dauer der Obdachlosigkeit der Person. Pionierstudien von Culhane und Kollegen (Kuhn und Culhane 1998) und ihre Wiederholungen in anderen Städten der Welt haben unterschiedliche Muster der Nutzung von Notunterkünften gezeigt, wobei zwischen langfristigen oder „chronischen“ Bewohnern von Notunterkünften mit Sucht- und psychischen Problemen, gelegentlichen oder episodischen Nutzern von Notunterkünften und einmaligen oder vorübergehenden Nutzern unterschieden wird.

Eine Vielzahl von sozial- und wirtschaftspolitischen Maßnahmen und Dienstleistungen kann versuchen, Obdachlosigkeit im Sinne von Prävention, Notfallmaßnahmen und Wiedereingliederungsmaßnahmen anzugehen. Im Mittelpunkt der „Notfallmaßnahmen“ für chronisch und episodisch wohnungslose Personen steht in vielen Ländern der „Housing First“- (oder allgemeiner oder wohnungsorientierter) Ansatz zur Bekämpfung von Obdachlosigkeit. Bei Housing-First-Ansätzen werden Wohnungen an wohnungslose Personen vermietet, ohne dass Bedingungen wie Nüchternheit oder Drogenfreiheit gefordert werden, und zwar grundsätzlich mit einem erheblichen Maß an Kontrolle über den Zeitpunkt und die Art der gleitenden Unterstützung, die sie erhalten (Pleace et al. 2019). Dies bezieht sich auf das dritte zentrale Forschungsergebnis, nämlich dass die meisten chronisch und episodisch wohnungslosen Personen mittels solcher Ansätze wie Housing First, die dauerhaft unterstütztes Wohnen anbieten, Wohnstabilität erreichen können. ${ }^{2}$ Umgekehrt können Menschen, die „vorübergehend“ obdachlos sind, die Nutzung von Notunterkünften aus eigener Initiative beenden (natürlich abhängig von günstigen Wohn-, Einkommensunterstützungs- und Arbeitsmarktbedingungen).

Abgesehen von den Vorteilen, die ein unabhängiges Leben für ehemals wohnungslose Menschen mit sich bringt, gibt es einen zusätzlichen Anstoß, von einer „Treppe des Übergangs“ (von der Straße über ein Wohnheim und eine betreute Unterkunft bis hin zum unabhängigen Wohnen) zu einem „Housing-First“-Modell der Dienstleistungsreaktion überzugehen, und den vielen negativen Merkmalen - wie Depersonalisierung und Hoffnungslosigkeit - von Notunterkünften (Finnerty 2018), besteht ein viertes Forschungsergebnis darin, die hohen Kosten von Notfallmaßnahmen bei Obdachlosigkeit zu benennen; diese ergeben sich, wenn man die Kosten für medizinische Notfallversorgung, Polizeieinsätze und andere Dienstleistungen zu den Kosten für die Bereitstellung von Unterkünften hinzurechnet (Parsell et al. 2017). ${ }^{3}$

\section{Ansätze zur Beteiligung der Nutzer}

bei Diensten für Wohnungslose

Die Bedeutung der Beteiligung von Dienstleistungsnutzern bei der Infragestellung paternalistischer Modelle der Sozialfürsorge hat in Bereichen wie der Gemeindeentwicklung und der psychischen Gesundheit einen wachsenden Einfluss, spielt aber im Bereich der Obdachlosigkeit kaum eine Rolle. Dennoch hat FEANTSA, der EU-weite Verband von Nichtregierungsorganisationen, die mit wohnungslosen Menschen arbeiten, ein Toolkit entwickelt, das von „Experten aus Erfahrung“ (aktuelle oder ehemalige Nutzer von Obdachlosendiensten) in Zusammenarbeit mit zehn nationalen Obdachlosenorganisationen erstellt wurde (FEANTSA, 2013). Das Toolkit liefert eine detaillierte Begründung für partizipative Ansätze und schlägt fünf Arten der Beteiligung vor: Information, Konsultation, Befragung der Dienstleistungsnutzer, Teilung der Macht und volle Kontrolle. ${ }^{4}$ - Volle Kontrolle bedeutet, dass obdachlose Dienstleistungsnutzer die Entscheidungsfindung kontrollieren, z. B. bei bestimmten Projekten, die vollständig von Dienstleistungsnutzern geleitet werden, aber innerhalb der Struktur einer Trägerorganisation verankert sind.

- Machtteilung bedeutet, dass die Nutzer von Diensten Entscheidungen mittragen, auch in der Verwaltung

- Einholung von Meinungen und Präferenzen der Dienstleistungsnutzer, z. B. durch Veranstaltungen für Interessenvertreter und Fokusgruppen

- Konsultation ist eine schwächere Form der Einholung von Nutzermeinungen und -präferenzen, mit geringerer Verbindlichkeit

- Information als geringste Form der Beteiligung - der Dienstleistungsnutzer erfährt durch Aushänge, Newsletters etc. von der Politik und den Dienstleistungen

Das Toolkit weist auch auf bestimmte Fallstricke partizipatorischer Ansätze in der Wohnungslosenhilfe hin, darunter die mögliche Alibifunktion, Konsultationsmüdigkeit und die Entstehung von „professionellen“ Dienstleistungsnutzern. ${ }^{5}$

FEANTSA (2013) definiert Partizipation als „eine Arbeitsweise, die Menschen befähigt, sich an Entscheidungen und Maßnahmen zu beteiligen, die ihr Leben betreffen. Sie basiert auf der Überzeugung, dass Menschen das Recht haben, mitzubestimmen, wie Dienstleistungen, die sie in Anspruch nehmen, eingerichtet und betrieben werden, und dass Menschen, die sozial oder wirtschaftlich benachteiligt sind, oft auf Barrieren stoßen, wenn es darum geht, Entscheidungen zu beeinflussen." (FEANTSA, 2013: 6). Durch Partizipation können Menschen auf persönlicher, sozialer und politischer Ebene mehr 


\section{Durchblick: Partizipation in der Wohnungslosenhilfe}

Empowerment erlangen: „Definitionen von Empowerment beinhalten die Idee, bestehende Machtannahmen in Frage zu stellen, Menschen dabei zu helfen, Kontrolle über ihr eigenes Leben zu erlangen und die Macht der Menschen zu fördern, damit sie sie in ihrem eigenen Leben nutzen können, indem sie sich für Themen einsetzen, die sie für wichtig halten ... Zusätzlich zum persönlichen Empowerment soll soziologisches Empowerment Mitglieder von Gruppen ansprechen, die durch soziale Diskriminierungsprozesse von Entscheidungsprozessen ausgeschlossen wurden." Andere potenzielle Ergebnisse von Partizipation sind Auswirkungen auf Dienstleistungen, auf die Politik und auf die Wahrnehmung eines breiten Spektrums von Zielgruppen, einschließlich der Ausbilder von sozialen Diensten und der allgemeinen Öffentlichkeit. Allerdings kann „Partizipation“ von Dienstleistern und Forschern recht unterschiedlich definiert werden: typischerweise (wie unten diskutiert) wird sie als „soziale Inklusion“ in Bereichen wie dem Arbeitsmarkt und dem sozialen Leben verstanden. FEANTSA (2013) schlägt 25 Dienstleistungsstandards für Partizipation in den Bereichen allgemeine Führung und Engagement, Praxis und Verfahren, Rekrutierung von und Umgang mit Nutzern, Training und Ressourcen und Evaluation vor, mit einer „Ampel“-Bewertung der Leistung jeder Klasse.

Die vorgeschlagenen Partizipationsinstrumente umfassen die gesamte Bandbreite der fünf partizipatorischen Ansätze, von Fragebögen und Fokusgruppen bis hin zu kreativer Kunst, „offenen Räumen“, Peer-Forschung, Peer-Bildung, Peer-Mentoring, Peer-Advocacy, Positionen in Komitees und Vorständen, Medienmöglichkeiten, Mitwirkung bei der Personalrekrutierung, von Nutzern geleitete Projekte, Pflegeplanung und -überprüfung und „Leading your own recovery“. Jedes Instrument wird beschrieben, seine Vor- und Nachteile aufgelistet und das Ausmaß der erforderlichen Ressourcen angegeben. Es ist erwähnenswert, dass FEANTSA den Schwerpunkt auf Notfälle und Wiedereingliederung legt und nicht auf präventive Dienste.

Ein aktuelles Beispiel für die Beteiligung von derzeit oder ehemals wohnungslosen Personen an der Interessenvertretung und Politikentwicklung ist die Initiative All in for Change! in Schottland. Unter der Leitung des Homeless Network Scotland, Cyrenians und Scottish Community Development Centre (SCDC), wird vorgeschlagen, „gelebte Erfahrungen in den Mittelpunkt des Systemwandels zu stellen, indem sie Mitarbeiter an vorderster Front und Menschen mit eigenen, persönlichen Erfahrungen mit Obdachlosigkeit zusammenbringt“. Die Gruppe will sich in die nationale Politikentwicklung einbringen und schlägt vor, ein schottlandweites
Netzwerk aufzubauen, um Politik und Praxis auf lokaler und nationaler Ebene zu beeinflussen (Homeless Network Scotland o.J.).

FEANTSA (2013) stellt fest, dass Dienstleistungsnutzer unterschiedliche Arten der Beteiligung an verschiedenen Dienstleistungen anstreben können. Dieser Punkt soll sich zwar auf Not- und Wiedereingliederungsdienste beziehen, gewinnt aber zusätzliche Kraft, wenn er mit der oben diskutierten ETHOS-Klassifizierung und mit der Unterscheidung zwischen vorübergehenden, episodischen und chronischen Dienstleistungsnutzern sowie zwischen Situationen mit hoher Obdachlosigkeit (in denen strukturelle Faktoren wie ungünstige Wohn- und Arbeitsmarktbedingungen vorherrschen) und Situationen mit geringer Obdachlosigkeit (in denen individuelle Merkmale vorherrschen) verknüpft wird. Die Schlüsselgruppen der Obdachlosen sind also, wie Whiteford (2011: 49) feststellt, „einige der verletzlichsten und isoliertesten Gruppen in der Gesellschaft mit begrenzter Kontrolle über die Organisation ihres Lebens.“

\section{Programme und Forschungen zum Thema Teilnahme der Nutzer von Diensten für Wohnungslose}

Die meisten veröffentlichten englischsprachigen Studien zur Partizipation wohnungsloser Dienstleistungsnutzer konzentrieren sich auf Not- und Wiedereingliederungsdienste für episodisch und chronisch wohnungslose Personen und nicht auf „Übergangs-“ oder "Risikopopulationen“. Ein Forschungsstrang, der die Partizipation durch die Linse „soziale Inklusion " erforscht, evaluiert die Auswirkungen spezifischer Programme zur Verbesserung von Bildungs-, Beschäftigungs- und anderen integrativen Programmen, die von Dienstleistern im Bereich der Wohnungslosenhilfe angeboten werden (Rutenfrans-Stupar et al. 2019; Johnston 2020). Ein anderer Ansatz besteht darin, Personen zu rekrutieren, die eine gemeinsame Erfahrung mit einem sozialen und gesundheitlichen Problem haben, wie z. B. Obdachlosigkeit in erster Linie, aber auch zusätzliche Merkmale je nach den Umständen (z. B. Erfahrung mit institutionellen Settings wie Pflege, Gefängnis, Streitkräfte oder mit geschlechtsspezifischer Gewalt), und die Unterstützung für Menschen, die gerade erst wohnungslos geworden sind oder solche, die versuchen, aus der Obdachlosigkeit herauszukommen, über eine Vielzahl von Diensten und Settings hinweg anzubieten. Diese Form der Teilnahme als „Experten aus Erfahrung“ kann auch bei der Ausbildung von professionellem Personal in sozialen Diensten, wie z. B. Sozialarbeitern, und bei der Peer-Forschung helfen. 
In Anbetracht der typischerweise komplexen Bedürfnisse dieser Bevölkerungsgruppe deckt ein Großteil der Forschung aus dem breiten Spektrum der oben diskutierten Möglichkeiten nur ein enges Spektrum von Peer Education, Peer Mentoring, Betreuungsplanung ab. Dies kann durch eine Reihe von oben erwähnten Faktoren geprägt sein, wie z. B. den Übergangscharakter der Obdachlosigkeit bei vielen Dienstleistungsnutzern. ${ }^{6}$ Effektiv führt dies zu einem stärker individualisierten und „therapeutischen “ Forschungsansatz für die Teilnahme in diesen Settings. Dies kann schnell durch das Übergewicht solcher Studien belegt werden, die von akademischen Suchmaschinen als Antwort auf relevante Schlüsselwörter zurückgegeben werden. Es findet sich ein überwältigender Fokus auf z. B. Peer-Unterstützung für wohnungslose Personen, die auch „medizinisch gefährdet" sind (Bean et al. 2013), mit problematischem Drogen- oder Alkoholkonsum (Parkes et al. 2019) oder an PTBS leiden (Van Voorhees et al. 2019).

In Anbetracht der Vielfalt und Komplexität dieses sozialen Problems und der betroffenen Gruppen können andere Peer-geleitete pädagogische Interventionen Kinder in obdachlosen Familien ansprechen, z. B. diskutieren Bradley et al. (2020) eine Peer-geleitete Elternintervention für obdachlose Familien in London; andere Studien richten sich auf Schadensreduzierung bei Drogenkonsum und riskantem Sexualverhalten (Barman-Adhikari et al. 2017), oder die Nutzung von psychischen Gesundheitsdiensten durch obdachlose Frauen (Duke und Searby 2019).

Aktuelle oder ehemals wohnungslose Expert_innen aus Erfahrung können auch zur Bildungsbildung von Fachkräften der Sozialen Arbeit beitragen. Im Kontext der Debatten in der Sozialen Arbeit über Machtungleichgewichte und Erfahrungsdefizite von Sozialarbeiter_innen gegenüber ihren Klient_innen diskutieren Geregová und Frišaufová (2020) ein Modul der Sozialen Arbeit an der Masayrk-Universität in Brünn, das die Teilnahme von Langzeit-Wohnungslosen mit einer Vorgeschichte von Drogenmissbrauch beinhaltete.

\section{Schlussfolgerungen}

Ein Großteil der verfügbaren (englischsprachigen) Forschung deckt aus der oben diskutierten breiten Palette von Partizipationsmöglichkeiten einen etwas engeren Bereich von Peer Education, Peer Mentoring und Care Planning ab. Effektiv führt dies zu einem eher individualisierten und „therapeutischen“ Forschungsansatz zur Partizipation in diesen Settings. Es ist unklar, ob dies eine enge Auslegung der in FEANTSA (2013) beschriebenen Partizipationsmöglichkeiten widerspiegelt, oder ob eine breitere Praxis der Partizipation in der akade- mischen Forschung nicht erfasst wird (oder in relativ unzugänglicher „grauer Literatur“ verbleibt). Es kann jedoch auch die Hypothese aufgestellt werden, dass partizipative Initiativen - ob eng oder weit gefasst - die Bedürfnisse von episodisch und chronisch wohnungslosen Personen mit komplexen Bedürfnissen in Not- und Wiedereingliederungsdiensten im Fokus haben.

Der Beitrag wurde von Peter Herrmann übersetzt.

Eingegangen. 15. Dezember 2020

Angenommen. 14. Januar 2021

1. Die Übersetzung aus dem Englischen unter https://www.feantsa. org/download/ethos_de_2404538142298165012.pdf 21-01-24.

2. Die Forschungsergebnisse sind jedoch uneinheitlicher in Bezug auf die Auswirkungen von Housing-First-Ansätzen auf Dimensionen wie psychische Gesundheit oder soziale Eingliederung. Siehe National Academies of Sciences Engineering and Medicine (2018) und Keenan et al. (2020). Eine nützliche Ressource für Dienstleistungsanbieter ist der Housing First Europe Hub unter https:// housingfirsteurope.eu/.

3. $\mathrm{Zu}$ diesen breiteren sozialen Kosten könnten nun die Kosten für die öffentliche Gesundheit während der Pandemie hinzukommen. Pawson et al. (2020: 61) weisen in einer Überprüfung der australischen Politik darauf hin, dass „[w]enn wir über die Vorteile berichten, die viele wichtige COVID-19-Maßnahmen für Menschen mit Obdachlosigkeit darstellen, müssen wir anerkennen, dass diese politischen und praktischen Maßnahmen das Ziel hatten, primär der Gesellschaft, nicht aber den Obdachlosen zu nutzen."

4. Für andere Typologien der Beteiligung von Dienstleistungsnutzern siehe Bradfield und Eckersley (2007) sowie Phillips und Kuyini (2018).

5. Dies deckt sich mit einigen der untersten Sprossen von Arnsteins bekanntem Rahmenwerk „Leiter der Beteiligung“ (Bradfield und Eckersley 2007).

6. Bradley et al. (2007: 24) stellen fest, dass ,es viel schwieriger ist, ein Engagement mit Personen zu erreichen, die nicht obne weiteres in eine organisierte Gruppe passen oder die sich entscheiden, sich überhaupt keiner Gruppe anzuschließen. Es wurde festgestellt, dass es schwierig ist, sich mit Personen zu beraten, die z. B. schwimmende Unterstützung erhalten, da sie sich nicht unbedingt untereinander treffen oder in ein organisiertes Kollektivorgan passen".

\section{Literatur}

Barman-Adhikari, A., Hsu, H., Begun, S., Portillo, A. P., \& Rice, E. (2017). Condomless sex among homeless youth: the role of multidimensional social norms and gender. AIDS and Behavior, 21(3), 688-702.

Bean, K. F., Shafer, M. S., \& Glennon, M. (2013). The impact of housing first and peer support on people who are medically vulnerable and homeless. Psychiatric Rehabilitation Journal, 36(1), 48-50. https:// doi.org/10.1037/h0094748.

Bradfield, H., \& Eckersley, T. (2007). Service user involvement. Reaching the hard to reach in supported housing. London: Jessica Kingsley. 


\section{Durchblick: Partizipation in der Wohnungslosenhilfe}

Bradley, C., Day, C., Penney, C., \& Michelson, D. (2020). 'Every day is hard, being outside, but you have to do it for your child': Mixed-methods formative evaluation of a peer-led parenting intervention for homeless families. Clinical Child Psychology and Psychiatry, 25(4), 860-876.

Bramley, G., \& Fitzpatrick, S. (2018). Homelessness in the UK: who is most at risk? Housing Studies, 33(1), 96-116.

Busch-Geertsema, V., Edgar, B., O’Sullivan, E., \& Pleace, N. (2010). Homelessness and homeless policies in Europe: lessons from research. Brussels: European Commission.

Daly, M. (2019). ESPN Thematic Report on National strategies to fight homelessness and housing exclusion-Ireland. Brussels: European Commission.

Duke, A., \& Searby, A. (2019). Mental ill health in homeless women: a review. Issues in Mental Health Nursing, 40(7), 605-612.

Edgar, W., Doherty, J., \& Meert, H. (2003). Second review of statistics on homelessness in Europe. Brussels: FEANTSA.

FEANTSA (2013). Participation Toolkit. Brussels: FEANTSA.

Finnerty, J. (2018). Last resort. Cork: Cork Simon Community.

Fitzpatrick, S. (2005). Explaining homelessness: a critical realist perspective. Housing, Theory and Society, 22(1), 1-17.

Geregová, M., \& Frišaufová, M. (2020). People with experience of longterm drug use and homelessness teaching with us: experts by experience participation in university social work education. Social Work Education, 39(3), 315-328.

Homeless Network Scotland Are you all in for change? eht-all-in-forchange-2.pdf. homelessnetwork.scot

Johnston, S. (2020). A working life: the continuing journey. Cork: Cork Simon Community.

Keenan, C., Miller, S., Hanratty, J., Pigott, T., Hamilton, J., \& Coughlan, C. (2020). Accommodation-based programmes for individuals experiencing or at risk of homelessness: a systematic review andnet work meta-analysis. London: Centre for Homelessness Impact. https://www.homelessnessimpact.org/

Kuhn, R., \& Culhane, D. (1998). Applying cluster analysis to test a typology of homelessness by pattern of shelter utilization: results from the analysis of administrative data. American Journal of Community Psychology, 26(2), 207-232.

National Academies of Sciences Engineering and Medicine (2018). Evidence of effect of permanent supportive housing on health. In Permanent supportive housing: evaluating the evidence for improving health outcomes among people experiencing chronic homelessness (S. 38-57). Washington, DC: The National Academies Press.

Parkes, T., Matheson, C., Carver, H., Budd, J., Liddell, D., Wallace, J., Pauly, B., Fotopoulou, M., Burley, A., Anderson, I., Maclennan, G. \& Foster, R. (2019). Supporting Harm Reduction through Peer Support (SHARPS): testing the feasibility and acceptability of a peer-delivered, relational intervention for people with problem substance use who are homeless, to improve health outcomes, quality of life and social functioning and reduce harms: study protocol. Pilot and Feasibility Studies, 5.

Parsell, C., Petersen, M., \& Culhane, D. (2017). Cost offsets of supportive housing: evidence for social work. The British Journal of Social Work, 47(5), 1534-1553.

Pawson, H., Parsell, C., Liu, E., Hartley, C., and Thompson, S. (2020). Australian Homelessness Monitor. Victoria: Launch Housing.

Peace, N., Baptista, I. \& Knutagård, M. (2019). Housing First in Europe - An Overview of Implementation, Strategy and Fidelity. Brussels and Helsinki: Housing First Europe Hub.

Phillips, D., \& Kuyini, A. B. (2018). Consumer participation at specialist homelessness services: do the homeless have a say in the services they receive? International Social Work, 61(6), 1095-1115.

Rutenfrans-Stupar, M., Van Der Plas, B., Den Haan, R., Van Regenmortel, T. \& Schalk, R. (2019). How is participation related to well-being of homeless people? An explorative qualitative study in a Dutch homeless shelter facility. Journal of Social Distress and Homelessness, 28(1), 44-55.

Stephens, M., Fitzpatrick, S., Elsinga, M., van Steen, G. \& Chzhen, Y. (2010). Study on Housing Exclusion: Welfare Policies, Housing Provision and Labour Markets. Brussels: European Commission, Directorate-General for Employment, Social Affairs and Equal Opportunities.
Van Voorhees, E. E., Resnik, L., Johnson, E., \& O'Toole, T. (2019). 'Posttraumatic stress disorder and interpersonal process in homeless veterans participating in a peer mentoring intervention: Associations with program benefit'. Psychological Services, 16(3), 463-474.

Whiteford, M. (2011). Square Pegs, Round Holes: Rough Sleeping and Service User Involvement? Practice, 23(1), 45-58.

Wright, J., Rubin, B. A., \& Devine, J. (1998). Beside the Golden Door. New York: Aldine De Gruyter. 